\title{
Effects of serotonin depletion and dopamine depletion on bimodal divided attention
}

\author{
Königschulte, W.1,2, Civai, C. ${ }^{3}$, Hildebrand, P.1,2, Gaber, T.J. ${ }^{1,2}$, Fink, G.R. ${ }^{4,5}$, Zepf, F.D. ${ }^{1,6,7,8}$ \\ ${ }^{I}$ Clinic for Child and Adolescent Psychiatry, Psychosomatics and Psychotherapy, \\ RWTH Aachen University, Aachen, Germany \\ ${ }^{2}$ JARA Translational Brain Medicine, Aachen \& Jülich, Germany \\ ${ }^{3}$ School of Psychology, University of Kent, Canterbury, UK \\ ${ }^{4}$ Cognitive Neuroscience, Institute of Neuroscience and Medicine (INM-3), \\ Research Centre Jülich, Jülich, Germany \\ ${ }^{5}$ Dept. of Neurology, University of Cologne, Germany \\ ${ }^{6}$ Clinic for Child \& Adolescent Psychiatry, Psychosomatics and Psychotherapy, \\ Friedrich Schiller University, Jena, Germany \\ ${ }^{7}$ Centre and Discipline of Child and Adolescent Psychiatry, Psychosomatics and Psychotherapy, \\ School of Medicine, Division of Psychiatry and Clinical Neurosciences \& Division of Paediatrics and Child \\ Health, Faculty of Medicine and Health Sciences, The University of Western Australia, Perth, Australia \\ ${ }^{8}$ Telethon Kids Institute, Perth, Australia
}

\section{Corresponding author:}

Chair and Winthrop Professor Dr med. Florian Daniel Zepf

Centre and Discipline of Child and Adolescent Psychiatry,

Psychosomatics and Psychotherapy

The University of Western Australia

35 Stirling Highway (M561)

Perth WA 6009

Australia

Email: florian.zepf@uwa.edu.au 


\section{ABSTRACT}

Objective: This study aimed to explore the effects of acute phenylalanine-tyrosine depletion (APTD) and acute tryptophan depletion (ATD) on bimodal divided attention. A balanced amino acid mixture (BAL) served as control condition.

Methods: 54 healthy adults (age: $\mathrm{M}=23.8$ years) were randomly assigned to APTD, ATD or BAL in a double-blind, between-subject approach. Divided attention was assessed after 4 hours. Blood samples were taken before and 6 hours after challenge intake.

Results: Amino acid concentrations following challenge intake significantly decreased (all $p$ $\leq .01)$. There was a significant difference in the mean reaction time (RT) towards auditory stimuli, but not towards visual stimuli between the groups. Post-hoc comparison of mean RT's (auditory stimuli) showed a significant difference between ATD $(\mathrm{RT}=604.0 \mathrm{~ms}, \mathrm{SD}=$ $56.9 \mathrm{~ms})$ and APTD $(\mathrm{RT}=556.4 \mathrm{~ms}, \mathrm{SD}=54.2 \mathrm{~ms} ; p=.037)$, but no RT-difference between $\mathrm{ATD}$ and $\mathrm{BAL}$ or APTD and $\mathrm{BAL}(\mathrm{RT}=573.6 \mathrm{~ms}, \mathrm{SD}=45.7 \mathrm{~ms})$.

Conclusion: The results indicate a possible dissociation between the effects of a diminished brain 5-HT and DA synthesis on the performance in a bimodal divided attention task. The difference was exclusively observed within the RT towards auditory signals.

Key words: Acute tryptophan depletion, phenylalanine tyrosine depletion, serotonin, dopamine, attention, healthy volunteers 


\section{INTRODUCTION}

The ability to allocate attention simultaneously to concurrent stimuli is essential for many daily life activities. Aberrant performance in this particular attentional domain is considered to be a pathognomonic hallmark in patients with, but not limited to, schizophrenia (Allen 1982; Ahlers et al. 2014; Shen et al. 2014), major depressive disorder (Thomas et al. 1998; Majer et al. 2004) and attention deficit hyperactivity disorder (Macleod and Prior 1996). Accordingly, a thorough understanding of the biochemical processes involved in divided attention is mandatory. The biogenic amines serotonin (5-HT) and dopamine (DA) are known to play a crucial role in the regulation of mood and cognitive processes (Robbins 2005; Ruhé et al. 2007), and dysfunction of these neurotransmitter systems is believed to be critical in the pathogenesis of the mentioned psychiatric disorders.

Experimental manipulation of brain DA and 5-HT systems allows to examine the contribution of these neurotransmitter systems to the neurochemical processes underlying attention.. Therefore, dietary neurotransmitter precursor depletion techniques such as acute tryptophan depletion (ATD, which leads to a decreased central nervous synthesis rate of 5-HT) or acute phenylalanine tyrosine depletion (APTD, which decreases DA synthesis in the brain) have become favorable tools in neuropsychological research (Booij et al. 2003; Young 2013; Biskup et al. 2015).

The two neurodietary procedures ATD and APTD build on the fact that the amino acid (AA) tryptophan (TRP) is the physiological precursor of 5-HT, and that AAs phenylalanine (PHE) and tyrosine (TYR) serve as essential substrates for central nervous system DA synthesis (Moja et al. 1988; Moja et al. 1996; Sheehan et al. 1996; Young 2013). Given that both 5-HT and DA cannot overcome the blood brain barrier, the formation of these monoamines depends 
on the availability of their respective physiological precursors, which belong to the group of large neutral amino acids (LNAAs), in the central nervous system. All LNAAs compete for the same transporter system at the blood brain barrier (Oldendorf and Szabo 1976), whereas the amount of TRP or PHE entering the brain by passive diffusion is negligible under physiological conditions (Momma et al. 1987; Smith et al. 1987).

Precursor influx is determined by the ratio of the respective AAs to the competing LNAAs (see for example Dingerkus et al. 2012). Enzymes related to the rate-determining step of central nervous 5-HT- and DA-synthesis are not saturated under physiological conditions (Carlsson and Lindqvist 1978; Young and Gauthier 1981), and a reduction in this particular ratio decreases central nervous 5-HT and/or DA synthesis. Depletion of 5-HT and DA in the central nervous system therefore can occur via diminished precursor availability due to a decreased dietary AA intake and dietary administration of competing LNAAs, whereby the latter also stimulates protein biosynthesis, so that preexisting precursor AAs are withdrawn from circulation (Moja et al. 1991). The significance of the ensuing effects on central monoamine turnover has been documented both in animal and human studies (Booij et al. 2003; Ruhé et al. 2007; Mendelsohn et al. 2009; Biskup CS et al. 2012; Young 2013; Sanchez et al. 2014). As demonstrated for the ATD protocol a significant reduction of the TRP influx occurs 90 minutes after ATD intake and reaches a nadir at 240 minutes, which is followed by a slow recuperation, whereas the TRP influx is still decreased at about $50 \%$ at 360 minutes after intake (Linden et al. 2016), Dingerkus et al. 2012).

Studies in animals and humans provide broad evidence for a serotonergic influence on dopaminergic neurotransmission. Early findings suggested a general opponency between both neurotransmitters systems, however, recent studies have indicated that also facilitating effects may occur (Daw et al. 2002; Cools et al. 2011). Hence, a more complex model including 
collaborative and opponent effects has been proposed (Boureau and Dayan 2011). The complex interaction of both systems is particularly apparent when the role of the prefrontal cortex (PFC) is examined. Playing a major role in attentional performance, the PFC is under the complex control of ascending monoaminergic projections, which in turn are under topdown control by the PFC (Robbins 2005). Functional and structural brain research identified the PFC as the fulcrum of cortico-striatal loops (Alexander et al. 1986; Robbins 2005). These frontostriatal systems are involved in a number of psychological processes intimately linked to executive functioning, like decision-making, selection and perception of novel information or behavioral control (Boulougouris and Tsaltas 2008).

There is a plenty of evidence that these circuits, which modulate executive functioning, are under serotonergic as well as dopaminergic influence, and that disturbances thereof are related to psychiatric disorders (Robbins 2005; Boulougouris and Tsaltas 2008). Due to its crucial importance to executive and attentional processing, Shallice and Norman considered the PFC as the neural substrate of the 'supervisory attentional system' (SAS) in their model of executive functioning of attentional control (Shallice 1982, 2004). The concept of a SAS was also included in the conceptual framework of attentional functioning provided by Zomeren and Brouwer (1994). The model by Zomeren and Brouwer regarding attention distinguishes between the selectivity and intensity of attention and proposed SAS as a modulating and controlling mechanism. Furthermore, the attention aspect of intensity was subdivided into "alertness" and "sustained attention", whereas attention selectivity was subdivided into "focused attention" and "divided attention".

To date, a limited number of experiments provided insight into the neural substrates of crossmodal divided attention. Experiments employing functional neuroimaging have highlighted the contribution of the dorsolateral PFC, as well as increased activation in regions such as the 
cingulate cortex or decreased activation in sensory cortices (Loose et al. 2003; Johnson and Zatorre 2005, 2006; Vohn et al. 2007; Chiang et al. 2013). However, less is known about the neurochemical underpinning of the concurrent allocation of attentional resources towards bimodal stimuli. The impact of ATD and APTD on attention has been investigated in several studies, although the majority of studies applied ATD instead of APTD, and only a few studies applied both (ATD and APTD). A review by (Mendelsohn et al. 2009) summarized the effects of ATD on memory, attention and executive functions. The authors concluded that there were mixed results, where sustained attention, divided attention or attentional setshifting were unaffected by ATD, while some studies reported improved performance regarding focused attention and an improved interference performance in a stroop task (Mendelsohn et al. 2009).

The published results on the effects of APTD on attention-related processes are also inconsistent. Scholes and colleagues reported improved attentional control in a stroop task after the administration of both ATD and APTD (Scholes et al. 2007), while VrshekSchallhorn (2006) et al observed impairments in a Go/No-Go paradigm after APTD administration. In contrast, Lythe and co-workers found no effect of APTD on Go/No-Go task performance as well as on set-shifting performance (2005). The latter findings are in line with the results provided by McLean et al. (2004) and Grevet et al. (2002). It is important to note that in the studies by Lythe, McLean and Grevet, the impact of APTD did not significantly differ from the impact of placebo on all aspects of attention investigated. To date, no studies exist that investigated the effects of APTD on performance in tests relating to divided attention in humans.

In this explorative study we used an APTD mixture dosed in accordance with body weight to reduce side effects, as this approach was already successfully employed by recent research in 
children and adolescents using ATD (Stadler et al. 2007; Kötting et al. 2013). Cross-modal divided attention performance as well as working memory performance, mood ratings and relevant plasma levels of different AAs were compared with an established body weight adapted ATD protocol and a balanced amino acid mixture (BAL) serving as a control condition. To the best of our knowledge, this is the first study to investigate the effects of a diminished central nervous synthesis of DA and 5-HT on bimodal divided attention and to provide data to calculate sample sizes for future research. A larger neuropsychological test battery was employed to assess, whether changes in bimodal attention were independent from other cognitive domains (e.g. mood), which are assumed to be related to dysfunction in these particular transmitter systems. 


\section{MATERIALS AND METHODS}

\section{Subjects and study design}

The study design was a randomized double-blind between-subject design, with the administration of ATD, APTD or BAL to healthy volunteers. Inclusion criteria were good physical health and the absence of any psychiatric or somatic disorder. Healthy adult volunteers of both genders were recruited and screened for psychiatric disorders by using a structured interview (SKID-light) (Demal 1999.). Key exclusion criteria were current or past history of any psychiatric or neurological disorder as defined in the ICD-10, primary somatic or any other chronic diseases, substance abuse, pregnancy in female subjects and an IQ lower than 85 . The study was assessed and approved by the local Ethics Committee, and was carried out in accordance with the Declaration of Helsinki. Written and oral informed consent was obtained from all participants prior to participation, and after the study subjects were financially compensated for their participation. In order to provide consistent environmental conditions, the study was carried out at the clinical research ward of the Jülich Research Centre. Participants were instructed to not consume alcohol the night before and on the day of the study, and to ensure sufficient sleep the night before the day of participation. All subjects were required to confirm that these instructions were followed prior to participation. After a 12-hour protein-fast, participants received a standardized low protein breakfast containing no TRP in the morning of the day of the investigation. Upon arrival at the study site, all subjects undertook a drug and pregnancy test (female subjects) before being randomly assigned to receive one of the three challenge conditions (ATD, APTD, BAL) in a double-blind, betweensubject approach. The neuropsychological assessment ended with an fMRI-task that was administered 300 minutes post intake (data from this experiment are the subject of a different publication). Serum and plasma samples were taken at two time points; before challenge intake $\left(\mathrm{T}_{0}\right)$, and after the last task of a neuropsychological testing battery $\left(\mathrm{T}_{6 \mathrm{~h}}\right)$. 


\section{Depletion procedure}

All AA compositions that were used in this study were based on the Moja-De protocol for acute tryptophan depletion (Zepf 2008). The AAs for the TRP balanced control condition (BAL) were dosed in accordance with the body-weight of the subjects and contained the following quantities (per $10 \mathrm{~kg}$ body weight): L-phenylalanine (1.32 g), L-leucine (1.32 g), Lisoleucine $(0.84 \mathrm{~g})$, L-methionine $(0.5 \mathrm{~g})$, L-valine $(0.69 \mathrm{~g})$, L-threonine $(0.6 \mathrm{~g})$, L-lysine (0.96 g, as 1,2g L-lysine-HCL) and L-tryptophan $(0,7 \mathrm{~g})$. The ATD (depletion of brain 5-HT synthesis) and APTD (depletion of brain DA synthesis) AA mixtures were deficient of the precursor TRP (ATD), and the precursors PHE and TRY (APTD) for the respective challenge procedures. In line with the dosage of the AAs of the BAL control condition, the relevant AAs of the ATD and APTD procedure (i.e., excluding the aforementioned precursors) used the same AA quantities as per $10 \mathrm{~kg}$ body weight. The mixtures were administered within an aqueous suspension as a beverage. Subjects were instructed to quickly consume the beverage.

\section{Neuropsychological assessment}

The computer-based Test of Attentional Performance Version 2.0 (TAP) was used to assess performance in divided attention (DV) and working memory (WM) four hours after challenge intake. Subjects were instructed to react to crucial stimuli as quickly as possible by pressing a button. Reaction time, omission and commission errors were registered. The working memory task used a 2-digit backward paradigm. Numbers from 1-9 were presented on screen in a putative arbitrary order. Subjects were required to respond when the presented number equaled the number that was presented two positions prior. The divided attention task required subjects to simultaneously react to a visual and auditory stimulus. Subjects were visually presented a $4 \times 4$ dot matrix that consisted of a varying number of crosses whilst being played a series of high and low pitched tones. Subjects were required to respond when four 
adjacent crosses formed a square and when a tone of the same pitch was presented consecutively.

\section{Mood assessment}

Throughout the study day, mood was assessed hourly using the ASTS, a brief German adaption of the Profile of Mood Scale (POMS). This self-rated questionnaire comprises 19 adjectives scored on a Lickert-scale ranging from 1 (not strong) to 7 (very strong). The ASTS measured subjective mood states on the five subscales "sadness," "hopelessness," "tiredness," “anger" and "positive mood". Negative emotion scores were calculated by summing up the first three subscales and the reversed values of the positive items.

\section{Laboratory assessment}

Venous blood samples were collected before and 6 hours after challenge intake. The tubes contained either Potassium-ETDA as anticoagulant to obtain plasma or silicate beads as clotting activator to obtain serum samples. The blood samples were stored at ambient temperature for $30 \mathrm{~min}$, and then centrifuged at $2.500 \mathrm{~g}$ for $10 \mathrm{~min}$. The supernatant was stored at $-80^{\circ} \mathrm{C}$ until laboratory analysis were carried out. TRP, PHE, TYR concentrations were measured using an immunosorbent assay (ELISA) kit, and concentrations of the competing BCAAs were analyzed using a commercially available enzyme BCAA test kit (Immundiagnostik AG, Bensheim, Germany, both).

\section{Data analysis}

Tukey's outlier test was applied, and normality of data was assessed using the Shapiro-Wilk Test. Due to the non-normal distribution of the data, which could not be corrected by conventional data transformation, plasma samples were analyzed using a non-parametric approach. Wilcoxon signed-rank tests were performed to investigate changes relative to 
baseline within the treatment groups. Kruskal-Wallis-Tests were used to evaluate differences between treatment groups. Post-hoc comparisons were carried out by Mann-Whitney Tests, the level of statistical significance was manually adjusted in accordance with Bonferroni $(\alpha=$ 0.017). One-way analyses of variance (ANOVA) were used to compare the results from the data derived by the TAP; Mood data were analyzed using a split-plot ANOVA. The level of statistical significance was set at $p=<0.05$. Because of the explorative nature of the study, the results were not subjected to alpha-adjustment. All statistical analyses were carried out using SPSS ${ }^{\circledR}$ for Microsoft Windows ${ }^{\circledR}$. As this study was conducted as a pilot study providing data to calculate sample sizes for future research, a post-hoc power analysis was carried out using $\mathrm{G}^{*}$ power $^{\circledR}$.

\section{RESULTS}

In total, $N=53$ subjects participated in the study, however after screening, the data of 4 subjects were excluded for various reasons, i.e. outliers in terms of unusually high error rates during the administered attention task, one subject in the APTD group was withdrawn from the study because of nausea approx. four hours after challenge-intake. This yielded a final sample of $\mathrm{N}=49$ subjects, the data of which were used for the final statistical analysis. The characteristics of the subjects in the final analyses are given in Table. 1.

(please insert Tab. 1 about here)

\section{Blood sample analysis}

The mean concentrations of all assessed AAs as well as the calculated AA ratios at each point for each treatment group are shown in Tab. 2 and Fig. 1. At baseline $\left(\mathrm{T}_{0}\right)$, no significant differences in plasma levels were observed between the three groups with respect to the 
relevant precursors $\left(\mathrm{TRP}_{\mathrm{T} 0} \chi^{2}(2, N=49)=1.50, p=.47\right.$; $\mathrm{PHE}$ т0 $\chi^{2}(2, N=49)=.59, p=.75$; $\left.\mathrm{TYR}_{\mathrm{T} 0} \chi^{2}(2, N=49)=.59, p=.74 ; \mathrm{BCCA}_{\text {т0 }} \chi^{2}(2, N=49)=.37, p=.83\right)$. Changes in plasma AA concentrations after challenge intake were in the expected direction (decrease), and these changes were found to be significant. A Kruskal-Wallis test followed by a Mann-Whitney-UTest indicated statistically significant differences in plasma levels between challenge treatment groups at time point $\mathrm{T}_{(6 \mathrm{~h})}\left(\mathrm{TRP}_{\mathrm{T} 6 \mathrm{~h}} \chi^{2}(2, N=49)=32.08\right.$; $\mathrm{PHE}_{\mathrm{T} 6 \mathrm{~h}} \chi^{2}(2, N=49)=$ 32.73; TYR T6h $\chi^{2}(2, N=49)=32.63, p<0.001$, all); TRP T6h: BAL vs. ATD $z=-4.82, p<.001$;

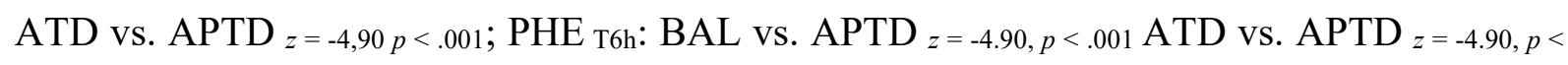
.001 ; TYR ${ }_{\text {T6h }}$ : BAL vs. APTD $z=-4.86, p<.001$; ATD vs. APTD $z=-4.90, p<.001$ ). Changes from baseline in plasma levels of TRP or TYR and PHE as well as the respective AA ratios were significant within treatment groups as determined by Wilcoxon signed-rank tests (ATD:

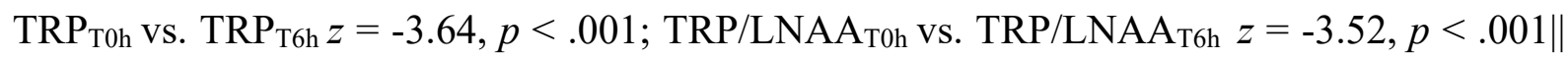
APTD: PHEт0h vs. PHET6h $z=-3.62, p<.001$; PHE/LNAAт0h vs. PHE/LNAAT6h $z=-3.62, p$ $<.001 ;$ TYR $_{\mathrm{T} 0 \mathrm{~h}}$ vs. TYR $\mathrm{T}_{6 \mathrm{~h}} z=-3.62, p<.001 ; \mathrm{TYR} \mathrm{LNAA}_{\mathrm{T} 0 \mathrm{~h}}$ vs. TYR/LNAA $\mathrm{T}_{6 \mathrm{~h}} z=-3.62$, $p<.001)$

(please insert Tab. 2 and Fig. 1 about here)

\section{Mood Assessment}

No significant changes from baseline in negative emotion scores or scores for tiredness were detected at the time of the neuropsychological assessment. There were no statistically significant time* group interactions $\left(\mathrm{F}_{(2,46)}=.44, \mathrm{p}=.65, \eta^{2}=.02\right)$ or main effects of time $(\mathrm{F}$ $\left.(1,46)=.22, \mathrm{p}=.65, \eta^{2}=.005\right) .($ Table 3$)$

(Fig. 2, Fig. 3, Tab. 3 and Tab. 4) 


\section{Neuropsychological assessment}

No significant group differences in reaction times $\left(\mathrm{F}_{(2,46)}=0.42, p=0.66\right)$, correct responses $\left(\mathrm{F}_{(2,46)}=0.08, \mathrm{p}=0.92\right)$ or omission error rates $\left(\mathrm{F}_{(2,46)}=0.08, p=0.92\right)$ were found in the working memory task (Tab. 4). A one-way ANOVA indicated a significant difference in the mean reaction time $(\mathrm{RT})$ towards auditory stimuli $\left(\mathrm{F}_{(2,46)}=3.46, p=0.04\right)$ but not towards visual stimuli between the challenge groups in the divided attention task. A Bonferroni posthoc comparison of the mean RT to auditory stimuli showed a significant difference between $\mathrm{ATD}(\mathrm{RT}=604.0 \mathrm{~ms}, \mathrm{SD}=56.9 \mathrm{~ms})$ and $\mathrm{APTD}(\mathrm{RT}=556.4 \mathrm{~ms}, \mathrm{SD}=54.2 \mathrm{~ms} ; p=.037)$ administration, but no difference in RT between ATD and BAL or APTD and BAL administration $(\mathrm{RT}=573.6 \mathrm{~ms}, \mathrm{SD}=45.7 \mathrm{~ms}$; Fig 1a/b). The one-way ANOVA of the reaction times toward auditory stimuli revealed a moderate to large effect size of 0.131 (partial eta squared). Based on this effect size a post-hoc power analysis revealed an overall statistical power $(1-\beta)$ of 0.66 .

\section{DISCUSSION}

To the best of our knowledge, this pilot study is the first that provides data regarding the effects of ATD and APTD on bimodal attentional performance in healthy adult participants. The results, albeit in a small sample size, indicate a possible dissociation between the effects of a short-term diminished central nervous 5-HT and DA synthesis on the performance in a bimodal divided attention task in healthy subjects. In particular, the difference was exclusively observed for reaction times towards auditory stimuli, whereas mean reaction time towards visual cues remained unaffected.

No significant group differences towards the balanced condition were observed, which may be due to the small sample size. Previous studies have not found effects of ATD on measures 
of divided attention, but the paradigms used in prior studies were not comparable to the present approach (Schmitt et al. 2000; Sobczak et al. 2002). However, the mean reaction time towards auditory stimuli in the balanced conditions was intermediate between the reaction times following ATD and APTD. This effect was irrespective of mood changes or wakefulness. The lack of fluctuation in mood observed in the present experiment is in line with previous findings indicating no alteration in mood following dietary monoamine depletion methods in healthy volunteers who do not have a history of an affective disorder (Ruhé et al. 2007). Moreover, no effects were observed on working memory, and - as has also been reported previously - both on phasic alertness and in a simple reaction task (Hildebrand et al. 2015). The former is of particular interest, regarding the proposed contribution of working memory to divided attention (Santangelo and Macaluso 2013).

In summary, it seems likely that the observed difference between the reaction times toward auditory stimuli is neither promoted by impairments in vigilance or motor response nor confounded by effects on working memory. Therefore, decreasing the central synthesis of dopamine and serotonin seems to play a role in the attentional performance towards auditory stimuli in a dual task paradigm. No definite conclusion can be drawn whether this apparent opponent effect emerges from the same neurophysiological level. Therefore the possible contributions of 5-HT and DA are discussed separately.

The administration of a TRP-free AA mixture resulted in a significant decrease in plasma levels of the particular AA and their calculated ratios. Decreases in total TRP levels were less distinct than in our previous findings and prior reports in literature (Mendelsohn et al. 2009). However, the change in the TRP-LNAA-ratio, a more valid estimator of the TRP-influx through the blood brain barrier, reached $65 \%$, which is comparable with previous findings. This suggests that the depletion magnitude in our experiment was sufficient to diminish 
central 5-HT synthesis, which is congruent with studies assessing depletion magnitude using nuclear imaging (Nishizawa et al. 1997), fenfluramine-prolactine-response (Coccaro et al. 1998) or cerebrospinal fluid metabolites measures (Williams et al. 1999). These results were also corroborated by an experiment in mice applying the identical depletion protocol (Sanchez et al. 2014). In the experiment by (Sanchez et al. 2014), ATD resulted in a marked reduction of the 5-HT turnover in the frontal cortex. This is of particular interest, given the relevance of the PFC with regard to the allocation of attentional resources.

To date, ATD has not been applied in experiments that utilized tasks of cross-modal divided attention. The neural substrates of cross-modal divided attention have been elucidated in a few functional imaging studies. Johnson et al. demonstrated that dividing attention between auditory and visual events resulted in an activation of the dorsolateral PFC (Johnson and Zatorre 2005, 2006). In a subsequent experiment, the authors showed that the ability to split attention deteriorated when there was a temporary disruption of dorsolateral PFC functioning (achieved through the use of transcranial magnetic stimulation) (Johnson et al. 2007). An fMRI study using the same attentional task revealed that splitting attention between visual and auditory stimuli resulted in an activation of the left PFC and the cingulate cortex as well as decreased activation in sensory brain areas (Loose et al. 2003). A study conducted by Vohn et al. observed activation in the right PFC, the right parietal cortex and the right claustrum under a cross modal divided attention paradigm (Vohn et al. 2007).

Overall, enhanced activity of the DLPFC and the ACC is fundamental in cross-modal divided attention tasks. Thus, the observed longer RT in the ATD group compared to those in the APTD group could be driven by modulation in these specific brain areas. A depressant effect of ATD on these brain areas has been shown in several imaging studies (Biskup et al. 2015). But aside from a SPECT study (Talbot and Cooper 2006) showing an attenuated regional 
cerebral blood flow in the left $\mathrm{dACC}$ following ATD, these effects were reserved to participants showing mood effects or were observed in the context of different stimuli.

A further explanation as to why the difference was exclusive to auditory cues could be the prominent role of 5-HT within the auditory system. Serotonergic neurons innervate the auditory system from cochlea to cortex (Hurley and Hall 2011). It has been shown that ATD modulates pre-attentive auditory change detection and early phases of auditory processing (Ahveninen et al. 2002; Ahveninen et al. 2003). As demonstrated at the subcortical level, the serotonergic input depends on the behavioral state (Hall et al. 2010; Hurley and Hall 2011). Therefore, under the demanding condition of a dual task, the auditory system could be especially prone to alteration in the serotonergic system.

The APTD resulted in a comparable depletion of the relevant AAs. The PHE-LNAA-ratio and the TYR-LNAA-ratio were decreased by $65 \%$ and $82 \%$, respectively. The depletion magnitude is comparable to previous studies (Booij et al. 2003). Notably, following the BAL and ATD treatment, the tyrosine plasma levels increased. Given that tyrosine was lacking in all AA compositions used in this experiment, this may be explained by a partial conversion of phenylalanine into tyrosine. Nevertheless, the increase of tyrosine occurred to a considerably lower extent compared to the increase of the LNAAs. Consequently, the TYR/LNNA ratios slightly declined both in the BAL and ATD condition when compared to the APTD condition.

At least at first sight, a faster reaction time under APTD relative to ATD may appear counterintuitive because dopaminergic stimulation is thought to improve attentional performance. Indeed, a large number of studies have provided evidence for this. However, optimal cognitive performance depends on an optimal level of DA. The relationship between optimal cognitive performance and levels of DA has been proposed to follow an "inverted u-shape", 
which is also dependent on the baseline behavioral state (Cools and D'Esposito 2011). Therefore, under specific conditions, a reduced dopaminergic tone may improve attentional performance as shown in experiment by Scholes et al (Scholes et al. 2007).

The findings of the current study show that dietary depletion techniques cannot be discussed from a one-dimensional quantitative perspective. Whilst former depletion studies targeted localized lesions or specific DA antagonists, dietary approaches decrease dopaminergic tones in a general manner and, as shown in animal studies, preferentially in specific cerebral regions. For instance, noradrenergic neurons are considered to be "immune" to dietary manipulation (Sheehan BD et al. 1996; McTavish et al. 1999). Therefore, it is likely that different kinds of dopaminergic neurons show different vulnerabilities towards a precursor deficiency. Additionally, DA itself exhibits different affinities to distinct receptor subtypes (Missale et al. 1998). Consequently, a decreased synaptic DA level affects different DA receptors in a divergent manner. Therefore APTD does not only result in an irregular reduction of dopaminergic functioning, but also in an enhanced DA efflux in specific neurocircuits. Inhibition of dopaminergic signaling by DA antagonists increases frontal dopaminergic function as has been shown by several neuropharmacological studies (Hernandez and Hoebel 1989; Liegeois et al. 2002).

An experiment using the same depletion technique in mice revealed a specific decrease in DA turnover in the striatum whereas the dopamine levels in the frontal cortex were not significantly affected (Sanchez et al. 2014). This is of particular interest, because a study in common marmosets showed that localized DA depletion of the striatum was associated with a greater focusing in a task requiring selective attention (Crofts et al. 2001). These results are in line with findings indicating that sulpirid enhances focusing in attentional tasks in humans (Mehta et al. 2004). Therefore, the relative variation in reaction times relative to ATD could 
be associated with improved prefrontal attentional control caused by a predominantly striatal dopamine depletion. This hypothesis is supported by human PET studies and animal experiments, indicating a predominant effect of ATPD on striatal structures (Mehta et al. 2005; Sanchez et al. 2014). Of note, APTD decreased striatal activity and increased activation in the PFC in an fMRI task related to reward processing (Frank et al. 2016). The improvements associated with APTD in task performance when compared to ATD may be caused by the modulation of cortico- striatal loops, whose functioning is crucial for attentional processes as mentioned introductorily. The modulation of the fronto-striato-circuitry by APTD has previously been studied by Scholes et al. in the context of reduced stroop interference under DA depletion (Scholes et al. 2007).

The collateral effects of this depletion method should also be considered. The intended decrease in the precursor efflux of the targeted monoamine concomitantly modulates the ratio of the relative neurotransmitter's precursors. Hence, ATD collaterally increases the availability of the DA precursors and APTD increases the availability of the 5-HT precursors. This is often considered as a limitation in depletion studies, although, the opponent effects that are attributable to DA and 5-HT functioning additionally underpin the hypothesis that the dissociation between the effects of a diminished central nervous 5-HT and DA synthesis are driven by an opponent DA-5HT interaction. Therefore it seems appropriate to conclude that a manipulation of the efflux determining AA ratio as achieved by ATD and APTD exerts opposing effects in the context of bimodal divided attention than solely referring to DA and 5HT availability. Due to the complex interplay of the various human metabolic pathways, additional effects of monoamine depletion cannot not be ruled out (Crockett et al., 2012; Zepf et al., 2013). For instance, the kynurenine pathways are affected by ATD (Kennedy et al. 2015). Several kynurenine metabolites can interact directly with central neural transmission (Schwarcz and Stone 2017), or indirectly e.g. by attenuating synthesis of tetrahydrobiopterin 
(Haruki et al. 2016), which can serve as a crucial co-factor for the biosynthesis of the 5-HT and DA (Sperner-Unterweger et al. 2014).

With regards to the translational value of such depletion experiments in different species, there is clear evidence that the depletion protocols used in the present study diminish 5HT/DA turnover in different strains of mice (Biskup et al. 2012; Sanchez et al. 2014). The particular translational value of such experiments conducted in rodents as done by Biskup et al. (2012) is that research in rodents allows highly invasive measurements that cannot be done in humans. In this particular study (Biskup et al. 2012), it was demonstrated that the Moja-De depletion paradigm decreased brain 5-HT in different brain areas, whereas influx kinetics as calculated for humans (see for example Dingerkus et al., 2012) can only be seen as an indirect measure or mathematical model of describing changed TRP-related influx kinetics after depletion across the blood brain barrier. Therefore, depletion protocols that can be used across different species have a great translational value, and because the Moja-De protocol can only be used in young people this particular depletion approach holds future promise to help to further disentangle the complex relationship between changes in TRP supply and influx, brain 5-HT synthesis and symptoms observed in patients with psychiatric disorders from a developmental viewpoint. With many psychiatric disorders emerging in childhood and adolescents, such investigations can help to understand the roles of tryptophan and 5-HT in psychiatric disorders in young people

A few limitations of the current study should be noted. These include the small sample size and the parallel design that could have lowered the statistical power. As calculated with $\mathrm{G}^{*}$ power $^{\circledR}$ a group size of at least 22 subjects would be necessary to reach a statistical power (1- $\beta$ ) of 0.8 . No gender-related analysis was carried because of the preliminary and explorative nature of this study, in particular because a control for the menstrual phase would 
have been valuable and this has been done in some previous studies (see Helmbold et al. 2016), but much ATD-related research has been done in males and the impact of the menstrual phase on ATD-research has often been neglected (Mendelsohn et al. 2009). Serotonergic neurotransmission shows significant gender differences and is influenced by sex hormones which alternate throughout the menstrual cycle (Lasiuk and Hegadoren 2007). Notably, plasma levels of TRP show also cyclic changes coinciding with gonatropine levels during the menstrual cycle (Carretti et al. 2005).

A separate execution of both tasks forming the dual task paradigm could also be considered in further studies. Additionally, this study was not able to assess whether the effect in the auditory condition would be present irrespective of the concomitant visual task. However, it seems likely that the current observations relating to the auditory condition would still stand, given the important role of 5-HT in the auditory system. Beside some limitations, this study sheds first light on a possible opponent modulation of a core attentional process via 5-HT and DA. However, further research is necessary to disentangle the interactions of 5-HT and DA in the context of divided attention. 


\section{CONFLICTS OF INTEREST/DISCLOSURE}

This study was funded by the Excellence Initiative of the German federal and state governments. The lead author (WK) was supported by the German Research Council (Deutsche Forschungsgemeinschaft DFG, IRTG 1328). FDZ was the recipient of an unrestricted award donated by the American Psychiatric Association (APA), the American Psychiatric Institute for Research and Education (APIRE) and AstraZeneca (Young Minds in Psychiatry Award). He has also received research support from the European Union, German Federal Ministry for Economics and Technology, the German Society for Social Pediatrics and Adolescent Medicine, the Paul and Ursula Klein Foundation, the Dr. August Scheidel Foundation, the IZKF fund of the University Hospital of RWTH Aachen University, the Telethon Perth Children's Hospital Research Fund (TPCHR); the Princess Margaret Foundation, and a travel stipend donated by the GlaxoSmithKline Foundation. He is the recipient of an unrestricted educational grant, travel support and speaker honoraria by Shire Pharmaceuticals, Germany. In addition, he has received support from the Raine Foundation for Medical Research (Raine Visiting Professorship), and editorial fees from Co-Action Publishing (Sweden). GRF serves as an editorial board member of Cortex, NeuroImage: Clinical, Zeitschrift für Neuropsychologie and Fortschritte der Neurologie Psychiatrie; receives royalties from the publication of the book Funktionelle MRT in Psychiatrie und Neurologie and Neurologische Differentialdiagnose; received honoraria for speaking engagements from TEVA, GlaxoSmith-Kline, Bayer, Desitin, Infoforum and Boehringer Ingelheim; and received or receives research support from the Bundesministerium für Bildung und Forschung and the Deutsche Forschungsgemeinschaft. The other authors have nothing to disclose and no conflict of interest. 


\section{REFERENCES}

Ahlers E, Hahn E, Ta TMT, Goudarzi E, Dettling M, Neuhaus AH. 2014. Smoking improves divided attention in schizophrenia. Psychopharmacology. 231(19):3871-3877.

Ahveninen J, Jaaskelainen IP, Pennanen S, Liesivuori J, Ilmoniemi RJ, Kahkonen S. 2003. Auditory selective attention modulated by tryptophan depletion in humans. Neurosci Lett. 340(3):181-184.

Ahveninen J, Kahkonen S, Pennanen S, Liesivuori J, Ilmoniemi RJ, Jaaskelainen IP. 2002. Tryptophan depletion effects on EEG and MEG responses suggest serotonergic modulation of auditory involuntary attention in humans. Neuroimage. 16(4):1052-1061.

Alexander GE, Delong MR, Strick PL. 1986. Parallel organization of functionally segregated circuits linking basal ganglia and cortex. Annu Rev Neurosci. 9:357-381.

Allen H. 1982. Dichotic monitoring and focused versus divided attention in schizophrenia. $\mathrm{Br}$ J Clin Psychol. 21(3):205-212.

Biskup C, Gaber T, Helmbold K, Bubenzer-Busch S, Zepf F. 2015. Amino acid challenge and depletion techniques in human functional neuroimaging studies: an overview. Amino acids. 47(4):651-683.

Biskup CS, Sanchez CL, Arrant A, Van Swearingen AE, Kuhn C, Zepf FD. 2012. Effects of acute tryptophan depletion on brain serotonin function and concentrations of dopamine and norepinephrine in C57BL/6J and BALB/cJ mice. PLoS One. 7(5):e35916. 
Booij L, Van der Does A, Riedel W. 2003. Monoamine depletion in psychiatric and healthy populations: review. Mol. Psychiatry. 8(12):951-973.

Boulougouris V, Tsaltas E. 2008. Serotonergic and dopaminergic modulation of attentional processes. Prog Brain Res. 172:517-542.

Boureau YL, Dayan P. 2011. Opponency Revisited: Competition and Cooperation Between Dopamine and Serotonin. Neuropsychopharmacology. 36(1):74-97.

Carretti N, Florio P, Bertolin A, Costa CV, Allegri G, Zilli G. 2005. Serum fluctuations of total and free tryptophan levels during the menstrual cycle are related to gonadotrophins and reflect brain serotonin utilization. Hum Reprod. 20(6):1548-1553.

Carlsson A, Lindqvist M. 1978. Dependence of 5-HT and catecholamine synthesis on concentrations of precursor amino-acids in rat brain. Naunyn Schmiedebergs Arch Pharmacol. 303(2):157-164.

Chiang TC, Liang KC, Chen JH, Hsieh CH, Huang YA. 2013. Brain deactivation in the outperformance in bimodal tasks: an FMRI study. PLoS One. 8(10):e77408.

Coccaro EF, Kavoussi RJ, Cooper TB, Hauger R. 1998. Acute tryptophan depletion attenuates the prolactin response to d-fenfluramine challenge in healthy human subjects. Psychopharmacology. 138(1):9-15. 
Cools R, D'Esposito M. 2011. Inverted-U-shaped dopamine actions on human working memory and cognitive control. Biol Psychiatry. 69(12):e113-125.

Cools R, Nakamura K, Daw ND. 2011. Serotonin and dopamine: unifying affective, activational, and decision functions. Neuropsychopharmacology. 36(1):98-113.

Crockett MJ, Clark L, Roiser JP, Robinson OJ, Cools R, Chase HW, Ouden Hd, ApergisSchoute A, Campbell-Meiklejohn D, Seymour B, Sahakian BJ, Rogers RD, Robbins TW. Converging evidence for central 5-HT effects in acute tryptophan depletion. Mol Psychiatry. 2012 Feb;17(2):121-3. doi: 10.1038/mp.2011.106. Epub 2011 Aug 30

Crofts HS, Dalley JW, Collins P, Van Denderen JCM, Everitt BJ, Robbins TW, Roberts AC. 2001. Differential effects of 6-OHDA lesions of the frontal cortex and caudate nucleus on the ability to acquire an attentional set. Cereb Cortex. 11(11):1015-1026.

Daw ND, Kakade S, Dayan P. 2002. Opponent interactions between serotonin and dopamine. Neural Netw. 15(4-6):603-616.

Demal U. 1999. SKIDPIT light - screening Bogen.

Dingerkus V, Gaber T, Helmbold K, Bubenzer S, Eisert A, Sánchez C, Zepf F. 2012. Acute tryptophan depletion in accordance with body weight: influx of amino acids across the bloodbrain barrier. J Neural Transm. 119(9):1037-1045. 
Frank S, Veit R, Sauer H, Enck P, Friederich HC, Unholzer T, Bauer UM, Linder K, Heni M, Fritsche A et al. 2016. Dopamine Depletion Reduces Food-Related Reward Activity Independent of BMI. Neuropsychopharmacology. 41(6):1551-1559.

Grevet EH, Tietzmann MR, Shansis FM, Hastenpflugl C, Santana LC, Forster L, Kapczinskil F, Izquierdo I. 2002. Behavioural effects of acute phenylalanine and tyrosine depletion in healthy male volunteers. J Psychopharmacol. 16(1):51-55.

Hall IC, Rebec GV, Hurley LM. 2010. Serotonin in the inferior colliculus fluctuates with behavioral state and environmental stimuli. J Exp Biol. 213(7):1009-1017.

Haruki H, Hovius R, Pedersen MG, Johnsson K. 2016. Tetrahydrobiopterin Biosynthesis as a Potential Target of the Kynurenine Pathway Metabolite Xanthurenic Acid. J Biol Chem. 291(2):652-657.

Helmbold K, Zvyagintsev M, Dahmen B, Biskup CS, Bubenzer-Busch S, Gaber TJ, Klasen M, Eisert A, Konrad K, Habel U et al. 2016. Serotonergic modulation of resting state default mode network connectivity in healthy women. Amino Acids. 48(4):1109-1120.

Hernandez L, Hoebel BG. 1989. Haloperidol Given Chronically Decreases Basal Dopamine in the Prefrontal Cortex More Than the Striatum or Nucleus Accumbens as Simultaneously Measured by Microdialysis. Brain Res Bull. 22(4):763-769.

Hildebrand P, Konigschulte W, Gaber TJ, Bubenzer-Busch S, Helmbold K, Biskup CS, Langen KJ, Fink GR, Zepf FD. 2015. Effects of dietary tryptophan and phenylalanine- 
tyrosine depletion on phasic alertness in healthy adults - A pilot study. Food Nutr Res. 59:26407.

Hurley LM, Hall IC. 2011. Context-dependent modulation of auditory processing by serotonin. Hearing Res. 279(1-2):74-84.

Johnson JA, Strafella AP, Zatorre RJ. 2007. The role of the dorsolateral prefrontal cortex in bimodal divided attention: Two transcranial magnetic stimulation studies. J Cognitive Neurosci. 19(6):907-920.

Johnson JA, Zatorre RJ. 2005. Attention to simultaneous unrelated auditory and visual events: Behavioral and neural correlates. Cereb Cortex. 15(10):1609-1620.

Johnson JA, Zatorre RJ. 2006. Neural substrates for dividing and focusing attention between simultaneous auditory and visual events. Neuroimage. 31(4):1673-1681.

Kennedy PJ, Allen AP, O'Neill A, Quigley EM, Cryan JF, Dinan TG, Clarke G. 2015. Acute tryptophan depletion reduces kynurenine levels: implications for treatment of impaired visuospatial memory performance in irritable bowel syndrome. Psychopharmacology (Berl). 232(8):1357-1371.

Kötting WF, Bubenzer S, Helmbold K, Eisert A, Gaber TJ, Zepf FD. 2013. Effects of tryptophan depletion on reactive aggression and aggressive decision-making in young people with ADHD. Acta Psychiatr Scand. 128(2):114-123. 
Lasiuk GC, Hegadoren KM. 2007. The effects of estradiol on central serotonergic systems and its relationship to mood in women. Biol Res Nurs. 9(2):147-160.

Liegeois JF, Ichikawa J, Meltzer HY. 2002. 5-HT2A receptor antagonism potentiates haloperidol-induced dopamine release in rat medial prefrontal cortex and inhibits that in the nucleus accumbens in a dose-dependent manner. Brain Res. 947(2):157-165.

Linden M, Helmbold K, Kempf J, Sippas S, Filss C, Langen KJ, Eisert A, Zepf FD. 2016. Dietary tryptophan depletion in humans using a simplified two amino acid formula - a pilot study. Food Nutr Res. 60:29272.

Loose R, Kaufmann C, Auer DP, Lange KW. 2003. Human prefrontal and sensory cortical activity during divided attention tasks. Hum Brain Mapp. 18(4):249-259.

Lythe KE, Anderson IM, Deakin JF, Elliott R, Strickland PL. 2005. Lack of behavioural effects after acute tyrosine depletion in healthy volunteers. J Psychopharmacol. 19(1):5-11. Macleod D, Prior M. 1996. Attention deficits in adolescents with ADHD and other clinical groups. Child Neuropsychology. 2(1):1-10.

Majer M, Ising M, Künzel H, Binder E, Holsboer F, Modell S, Zihl J. 2004. Impaired divided attention predicts delayed response and risk to relapse in subjects with depressive disorders. Psychol med. 34(08):1453-1463.

McLean A, Rubinsztein JS, Robbins TW, Sahakian BJ. 2004. The effects of tyrosine depletion in normal healthy volunteers: implications for unipolar depression. Psychopharmacology (Berl). 171(3):286-297. 
McTavish SF, Cowen PJ, Sharp T. 1999. Effect of a tyrosine-free amino acid mixture on regional brain catecholamine synthesis and release. Psychopharmacology (Berl). 141(2):182188.

Mehta MA, Gumaste D, Montgomery AJ, McTavish SF, Grasby PM. 2005. The effects of acute tyrosine and phenylalanine depletion on spatial working memory and planning in healthy volunteers are predicted by changes in striatal dopamine levels. Psychopharmacology (Berl). 180(4):654-663.

Mehta MA, Manes FF, Magnolfi G, Sahakian BJ, Robbins TW. 2004. Impaired set-shifting and dissociable effects on tests of spatial working memory following the dopamine D2 receptor antagonist sulpiride in human volunteers. Psychopharmacology (Berl). 176(3-4):331342.

Mendelsohn D, Riedel WJ, Sambeth A. 2009. Effects of acute tryptophan depletion on memory, attention and executive functions: a systematic review. Neurosci Biobehav Rev. 33(6):926-952.

Missale C, Nash SR, Robinson SW, Jaber M, Caron MG. 1998. Dopamine receptors: From structure to function. Physiol Rev. 78(1):189-225.

Moja E, Lucini V, Benedetti F, Lucca A. 1996. Decrease in plasma phenylalanine and tyrosine after phenylalanine-tyrosine free amino acid solutions in man. Life sciences. 58(26):2389-2395. 
Moja E, Restani P, Corsini E, Stacchezzini M, Assereto R, Galli C. 1991. Cycloheximide blocks the fall of plasma and tissue tryptohan levels after tryptohan-free amino acid mixtures. Life sci. 49(15):1121-1128.

Moja EA, Stoff DM, Gessa GL, Castoldi D, Assereto R, Tofanetti O. 1988. Decrease in plasma tryptophan after tryptophan-free amino acid mixtures in man. Life sci. 42(16):15511556.

Momma S, Aoyagi M, Rapoport SI, Smith QR. 1987. Phenylalanine transport across the blood-brain barrier as studied with the in situ brain perfusion technique. J Neurochem. 48(4):1291-1300.

Nishizawa S, Benkelfat C, Young SN, Leyton M, Mzengeza S, DeMontigny C, Blier P, Diksic M. 1997. Differences between males and females in rates of serotonin synthesis in human brain. P Natl Acad Sci USA. 94(10):5308-5313.

Oldendorf WH, Szabo J. 1976. Amino acid assignment to one of three blood-brain barrier amino acid carriers. Am J Physiol. 230(1):94-98.

Robbins TW. 2005. Chemistry of the mind: neurochemical modulation of prefrontal cortical function. J Comp Neurol. 493(1):140-146.

Ruhé HG, Mason NS, Schene AH. 2007. Mood is indirectly related to serotonin, norepinephrine and dopamine levels in humans: a meta-analysis of monoamine depletion studies. Mol psychiatry. 12(4):331-359. 
Sanchez CL, Van Swearingen AE, Arrant AE, Kuhn CM, Zepf FD. 2014. Dietary manipulation of serotonergic and dopaminergic function in $\mathrm{C} 57 \mathrm{BL} / 6 \mathrm{~J}$ mice with amino acid depletion mixtures. J Neural Transm (Vienna). 121(2):153-162.

Santangelo V, Macaluso E. 2013. The contribution of working memory to divided attention. Hum Brain Mapp. 34(1):158-175.

Schmitt JA, Jorissen BL, Sobczak S, van Boxtel MP, Hogervorst E, Deutz NE, Riedel WJ. 2000. Tryptophan depletion impairs memory consolidation but improves focussed attention in healthy young volunteers. J Psychopharmacol. 14(1):21-29.

Scholes KE, Harrison BJ, O'Neill BV, Leung S, Croft RJ, Pipingas A, Phan KL, Nathan PJ. 2007. Acute serotonin and dopamine depletion improves attentional control: findings from the stroop task. Neuropsychopharmacology. 32(7):1600-1610.

Schwarcz R, Stone TW. 2017. The kynurenine pathway and the brain: Challenges, controversies and promises. Neuropharmacology. 112(Pt B):237-24.

Shallice T. 1982. Specific Impairments of Planning. Philos T Roy Soc B. 298(1089):199-209. Shallice T. 2004. The Fractionation of Supervisory Control. In: Gazzaniga MS, editor.The cognitive neurosciences. Cambridge (MA), US: MIT Press; p. 943-956.

Sheehan B, Tharyan P, McTavish S, Campling G, Cowen P. 1996. Use of a dietary manipulation to deplete plasma tyrosine and phenylalanine in healthy subjects. J Psychopharmacol. 10(3):231-234. 
Sheehan BD, Tharyan P, McTavish SFB, Campling GM, Cowen PJ. 1996. Use of a dietary manipulation to deplete plasma tyrosine and phenylalanine in healthy subjects. J Psychopharmacol. 10(3):231-234.

Shen C, Popescu FC, Hahn E, Ta TT, Dettling M, Neuhaus AH. 2014. Neurocognitive pattern analysis reveals classificatory hierarchy of attention deficits in schizophrenia. Schizophr Bull. 40(4):878-885.

Smith QR, Momma S, Aoyagi M, Rapoport SI. 1987. Kinetics of neutral amino acid transport across the blood-brain barrier. J Neurochem. 49(5):1651-1658.

Sobczak S, Riedel WJ, Booij I, Aan Het Rot M, Deutz NE, Honig A. 2002. Cognition following acute tryptophan depletion: difference between first-degree relatives of bipolar disorder patients and matched healthy control volunteers. Psychol Med. 32(3):503-515.

Sperner-Unterweger B, Kohl C, Fuchs D. 2014. Immune changes and neurotransmitters: possible interactions in depression? Prog Neuropsychopharmacol Biol Psychiatry. 48:268-276 Stadler C, Zepf FD, Demisch L, Schmitt M, Landgraf M, Poustka F. 2007. Influence of rapid tryptophan depletion on laboratory-provoked aggression in children with ADHD. Neuropsychobiology. 56(2-3):104-110.

Talbot PS, Cooper SJ. 2006. Anterior cingulate and subgenual prefrontal blood flow changes following tryptophan depletion in healthy males. Neuropsychopharmacology. 31(8):17571767. 
Thomas P, Goudemand M, Rousseaux M. 1998. Divided attention in major depression. Psychiatry res. 81(3):309-322.

Vohn R, Fimm B, Weber J, Schnitker R, Thron A, Spijkers W, Willmes K, Sturm W. 2007. Management of attentional resources in within-modal and cross-modal divided attention tasks: An fMRI study. Hum Brain Mapp. 28(12):1267-1275.

Vrshek-Schallhorn S, Wahlstrom D, Benolkin K, White T, Luciana M. 2006. Affective bias and response modulation following tyrosine depletion in healthy adults. Neuropsychopharmacology. 31(11):2523-2536.

Williams WA, Shoaf SE, Hommer D, Rawlings R, Linnoila M. 1999. Effects of acute tryptophan depletion on plasma and cerebrospinal fluid tryptophan and 5-hydroxyindoleacetic acid in normal volunteers. J Neurochem. 72(4):1641-1647.

Young SN. 2013. Acute tryptophan depletion in humans: a review of theoretical, practical and ethical aspects. J Psychiatry Neurosci. 38(5):294-305.

Young SN, Gauthier S. 1981. Effect of tryptophan administration on tryptophan, 5hydroxyindoleacetic acid and indoleacetic acid in human lumbar and cisternal cerebrospinal fluid. J Neurol Neurosurg Psychiatry. 44(4):323-328.

Zepf FD, Sánchez CL, Biskup CS, Kötting WF, Bubenzer S, Helmbold K, Eisert A, Gaber TJ, Landgraf M, Dahmen B, Poustka F, Wöckel L, Stadler C, Grabemann M, Mette C, Heinrich V, Uekermann J, Abdel-Hamid M, Kis B, Zimmermann M, Wiltfang J, Kuhn CM. Acute tryptophan depletion - converging evidence for decreasing central nervous serotonin synthesis 
in rodents and humans. Acta Psychiatr Scand. 2014 Feb;129(2):157-9. doi: 10.1111/acps.12215. Epub 2013 Nov 18

Zepf FD. 2008. Untersuchung zentralnervöser serotonerger Funktionen mit Hilfe des »Rapid Tryptophan Depletion-Test« (RTD) bei männlichen Kindern und Jugendlichen mit Aufmerksamkeits-Defizit/Hyperaktivitäts Syndrom [Iinvestigation of central serotonergic functions in male children and adolescents with ADHD using the "Rapid Tryptophan Depletion test" (RTD)]. Marburg: Tectum Verlag.German.

Zomeren AHv, Brouwer WH. 1994. Clinical neuropsychology of attention. New York(NY): Oxford University Press. 
Tab. 1

\begin{tabular}{lcccc}
\hline & n & Weight $(\mathbf{k g} \pm$ SD) & Age $($ years \pm SD) & Gender $\mathbf{f} \mid \mathbf{m}$ \\
\hline BAL & 16 & $75,9 \pm 17,7$ & $26,4 \pm 5,9$ & $7 \mid 9$ \\
ATD & 16 & $71,2 \pm 13,5$ & $24,4 \pm 4,2$ & $8 \mid 8$ \\
APTD & 17 & $70,4 \pm 11,7$ & $23,6 \pm 3,1$ & $8 \mid 9$ \\
\hline
\end{tabular}

Legend to Tab. 1: Characteristics of the study sample (data are presented as mean values (M) +/- standard deviation (SD). 


\section{Tab.2}

\begin{tabular}{|c|c|c|c|c|c|}
\hline & & \multicolumn{2}{|l|}{$\mathbf{T 0}$} & \multicolumn{2}{|l|}{ T6h } \\
\hline & & Mean & SD & Mean & SD \\
\hline \multicolumn{6}{|c|}{ Phenylalanine } \\
\hline \multirow[t]{3}{*}[\mu\mathrm{mol}/\mathrm{L}]{} & BAL & 63,87 & 25,10 & 203,17 & 110,71 \\
\hline & ATD & 59,16 & 10,46 & 187,14 & 78,74 \\
\hline & PTD & 56,34 & 12,76 & $37,27 *$ & 11,89 \\
\hline \multicolumn{6}{|c|}{ Tryptophan } \\
\hline \multirow[t]{3}{*}[\mu\mathrm{mol}/\mathrm{L}]{} & BAL & 68,39 & 12,34 & 176,40 & 46,20 \\
\hline & ATD & 64,04 & 11,97 & $41,50 *$ & 16,89 \\
\hline & APTD & 67,84 & 10,01 & 204,42 & 68,39 \\
\hline \multicolumn{6}{|l|}{ Tyrosine } \\
\hline \multirow[t]{3}{*}[\mu\mathrm{mol}/\mathrm{L}]{} & BAL & 57,89 & 16,63 & 72,36 & 23,40 \\
\hline & ATD & 56,22 & 10,08 & 78,68 & 22,71 \\
\hline & APTD & 56,53 & 12,77 & $20,76 *$ & 8,21 \\
\hline \multicolumn{6}{|l|}{ BCCAs } \\
\hline \multirow[t]{3}{*}[\mu\mathrm{mol}/\mathrm{L}]{} & BAL & 413,61 & 89,70 & 670,58 & 134,06 \\
\hline & ATD & 397,30 & 104,93 & 719,8 & 175,71 \\
\hline & APTD & 384,06 & 64,09 & 740,38 & 116,54 \\
\hline \multicolumn{6}{|c|}{ Phenylalanine/ $\sum$ LNAA } \\
\hline & BAL & 0,12 & $\mathbf{0 , 0 3}$ & 0,23 & 0,13 \\
\hline & ATD & $\mathbf{0 , 1 2}$ & $\mathbf{0 , 0 2}$ & $\mathbf{0 , 2 3}$ & $\mathbf{0 , 1 1}$ \\
\hline & APTD & $\mathbf{0 , 1 1}$ & $\mathbf{0 , 0 2}$ & $0,04 *$ & $\mathbf{0 , 0 1}$ \\
\hline \multicolumn{6}{|c|}{ Tryptophan/ $\sum$ LNAA } \\
\hline & BAL & 0,13 & $\mathbf{0 , 0 3}$ & 0,19 & 0,04 \\
\hline & ATD & 0,13 & $\mathbf{0 , 0 3}$ & $0,05 *$ & $\mathbf{0 , 0 2}$ \\
\hline & APTD & 0,14 & $\mathbf{0 , 0 2}$ & 0,26 & $\mathbf{0 , 0 8}$ \\
\hline \multicolumn{6}{|c|}{ Tyrosine/ $\sum$ LNAA } \\
\hline & BAL & 0,11 & $\mathbf{0 , 0 2}$ & $\mathbf{0 , 0 7}$ & $\mathbf{0 , 0 3}$ \\
\hline & ATD & 0,11 & $\mathbf{0 , 0 2}$ & 0,08 & $\mathbf{0 , 0 2}$ \\
\hline & APTD & 0,11 & $\mathbf{0 , 0 2}$ & $\mathbf{0 , 0 2 *}$ & $\mathbf{0 , 0 1}$ \\
\hline
\end{tabular}

Legend to Tab. 2: The respective mean concentration \pm standard deviation (SD) of all assessed AAs as well as the calculated AA ratios at each time point for each treatment group. $(*=p<.001)$ 
Tab. 3

\begin{tabular}{|c|c|c|c|c|c|}
\hline & Mean ASTS & SD at & $\begin{array}{r}\text { ASTS } \\
\mathrm{T} \\
\end{array}$ & $\pm \mathrm{SD}$ at & \\
\hline \multicolumn{6}{|c|}{ "negative" } \\
\hline BAL & 44,31 & $\pm 9,87$ & 43,63 & $\pm 10,29$ & n.s. \\
\hline APTD & 41,41 & $\pm 5,59$ & 42,82 & $\pm 4,72$ & n.s. \\
\hline ATD & 41,44 & $\pm 6,43$ & 42,00 & $\pm 9,24$ & n.s. \\
\hline \multicolumn{6}{|l|}{ "tired" } \\
\hline BAL & 10,25 & $\pm 4,81$ & 8,81 & $\pm 5,09$ & n.s. \\
\hline APTD & 8,82 & $\pm 2,67$ & 8,29 & $\pm 3,67$ & n.s. \\
\hline ATD & 8,94 & $\pm 3,4$ & 8,06 & $\pm 4,15$ & n.s. \\
\hline
\end{tabular}

Legend to Tab. 3: Data are presented as the mean ASTS scores \pm standard deviation (SD). (n.s.: non-significant)

Tab. 4

\begin{tabular}{|cccccccc|}
\hline & BAL & \multicolumn{1}{c|}{ APTD } & & ATD & \\
& Mean & SD & Mean & SD & Mean & SD \\
$\begin{array}{c}\text { Correct } \\
\text { responses } \\
\text { (number) }\end{array}$ & 12,88 & 3,48 & 13,00 & 2,67 & 13,25 & 4,53 & n.s. \\
$\begin{array}{c}\text { Reaction time } \\
\text { (ms) }\end{array}$ & 564,69 & 114,98 & 604,82 & 128,93 & 600,50 & 162,00 & n.s. \\
$\begin{array}{c}\text { Omission } \\
\text { errors } \\
\text { (number) }\end{array}$ & 2,13 & 3,48 & 2,00 & 2,67 & 1,75 & 1,53 & n.s. \\
\hline
\end{tabular}

Legend to Tab. 3: Results of the working memory task presented as the respective means \pm standard deviation (SD). (n.s.: non-significant) 


\section{FIGURES}

Fig.1

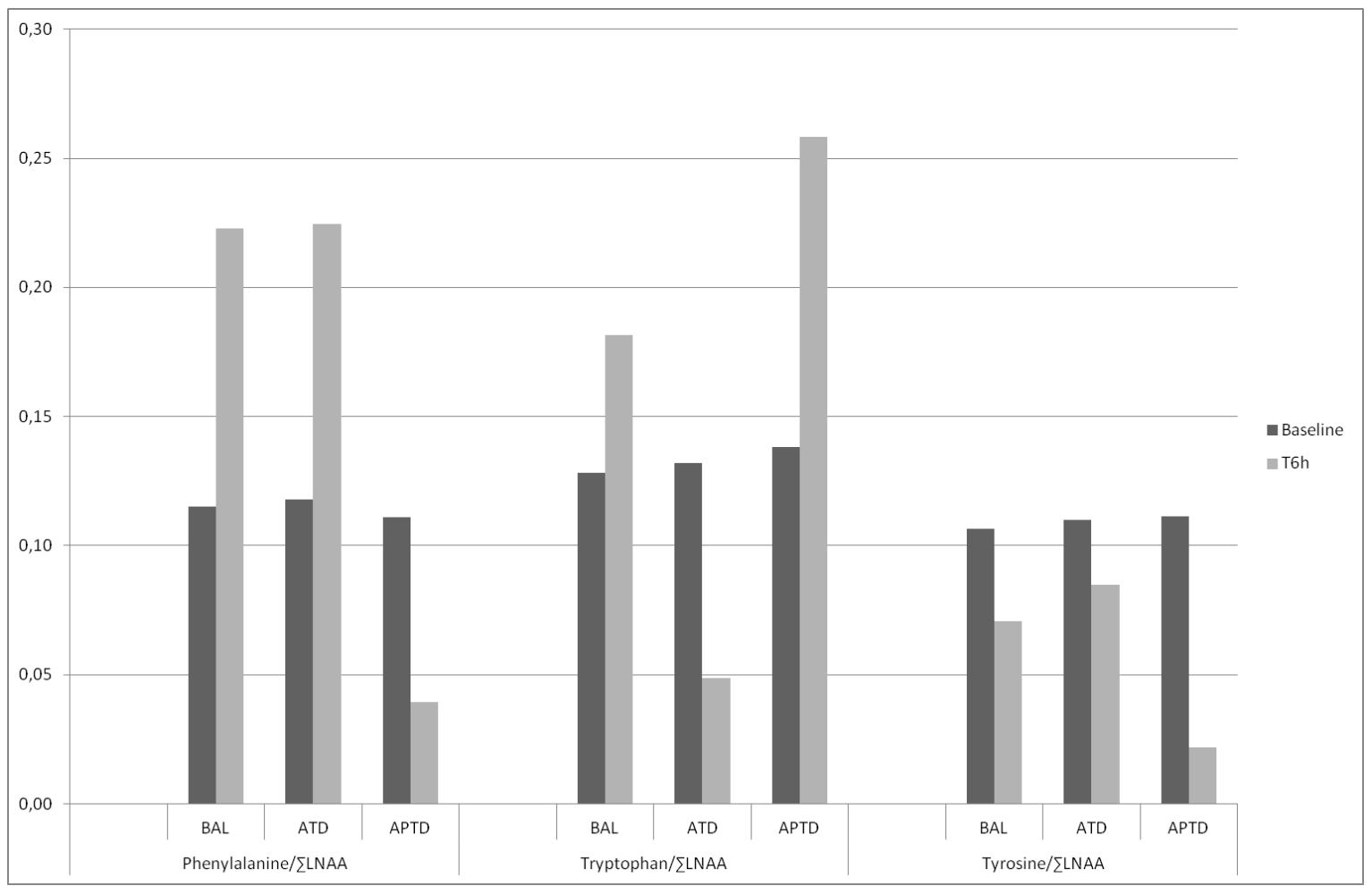

Legend to Fig. 1: The respective AA ratios at each time point for each treatment group 
Fig. 2

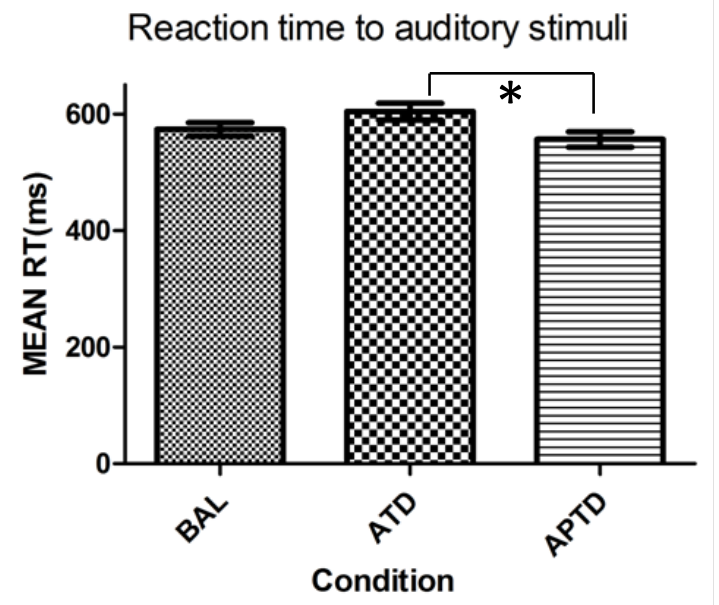

Legend to Fig. 2: Given are the mean reaction times to auditory stimuli for each condition. Error bars depict SEM. * $p<0.05$ 
Fig. 3

Reaction time to visual stimuli

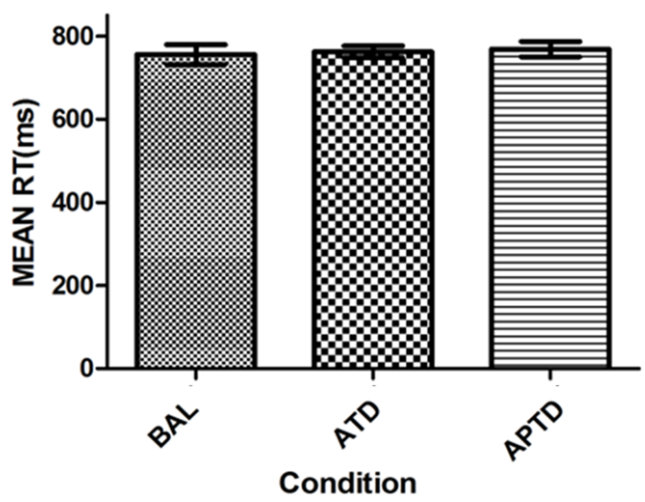

Legend to Fig. 3: Given are the mean reaction times to visual stimuli for each challenge condition. Error bars depict SEM. 